MRI-PHY/96-38

\title{
Phase Diagram of the Half-Filled Extended Hubbard Model in Two Dimensions
}

\author{
Biplab Chattopadhyay $^{\star}$ and D. M. Gaitonde ${ }^{\dagger}$ \\ The Mehta Research Institute of Mathematics \& Mathematical Physics, \\ 10 Kasturba Gandhi Marg, Allahabad 211002, INDIA
}

\begin{abstract}
We consider an extended Hubbard model of interacting fermions on a lattice. The fermion kinetic energy corresponds to a tight binding Hamiltonian with nearest neighbour $(-t)$ and next nearest neighbour $\left(t^{\prime}\right)$ hopping matrix elements. In addition to the onsite Hubbard interaction $(U)$ we also consider a nearest neighbour repulsion $(V)$. We obtain the zero temperature phase diagram of our model within the Hartree-Fock approximation. We consider ground states having charge and spin density wave ordering as well as states with orbital antiferromagnetism or spin nematic order. The latter two states correspond to particle-hole binding with $d_{x^{2}-y^{2}}$ symmetry in the charge and spin channels respectively. For $t^{\prime}=0$, only the charge density wave and spin density wave states are energetically stable. For non-zero $t^{\prime}$, we find that orbital antiferromagnetism (or spin nematic) order is stable over a finite portion of the phase diagram at weak coupling. This region of stability is seen to grow with increasing values of $t^{\prime}$.
\end{abstract}

PACS numbers: 71.27.+a, 71.28.+d, 71.35.+z

To appear in Phys. Rev. B (Brief Reports)

`Present email: biplab@hp1.saha.ernet.in, †email: dattu@mri.ernet.in ; 
The phase diagram of the 2-d Hubbard model has been a subject of intense study over the past few years, ever since the discovery of the high temperature superconductors. The strange properties of these materials are believed to arise because of strong electronic correlation and the 2-d Hubbard model or its strong coupling limit, the t-J model, have frequently been used as a starting point for theoretical attempts at understanding these phenomena. Even earlier, this model has been studied as the minimal model necessary for describing a host of phenomena ranging from metal-insulator transitions in the transition metal oxides to magnetism in itinerant electron systems.

On a square lattice, at half-filling, a tight-binding Hamiltonian with hopping restricted to nearest neighbours, has a Fermi surface that is perfectly nested, so that at zero temperature the ground state of the system is a two sublattice spin density wave (SDW) state for arbitrarily small onsite repulsion (U). Detailed studies [1] of the SDW Hartree-Fock (HF) solutions as well as a random-phase-approximation (RPA) study of the collective excitation spectrum have shown that this approach is effective even at large values of $U$ where the magnetization and spin wave dispersion obtained from a mapping to the Heisenberg model are recovered. The introduction of a nearest-neighbour repulsion $(\mathrm{V})$ introduces a tendency towards charge ordering in the form of a charge density wave $(\mathrm{CDW})$ and for sufficiently large values of $\mathrm{V}(V>U / 4)$, the CDW state becomes energetically preferred to the SDW state.

It has been known since the early work of Halperin and Rice [2] that there exist forms of particle-hole ordering other than the CDW and SDW state. In this context, some other ground states which have been considered are orbital antiferromagnetism $(\mathrm{OAF})$ and spin nematic (SN) [3-6]. These ground states can be thought of as arising from d-wave pairing in the particle-hole charge and spin channels respectively [7]. The $\mathrm{OAF}$ and SN states have charge and spin currents in every elementary plaquette of the square lattice with neighbouring plaquettes having their currents oriented in opposite directions. These states are thus similar to the staggered flux phases which were studied earlier [8] as possible ground states of the t-J model, in the strong correlation limit.

In this paper we present a Hartree-Fock study of the zero temperature phase diagram of the extended Hubbard model at half-filling on a square lattice especially focussing on the competition of the CDW and SDW states with the OAF or SN states. Our model has a kinetic energy corresponding to nearest-neighbour and next nearest-neighbour hopping 
whose amplitudes are $t$ and $t^{\prime}$ respectively and an onsite (U) and nearest neighbour (V) repulsive interactions. For $t^{\prime}=0$ we find that the CDW and SDW states are the only stable ground states of the system and are separated by a phase boundary at $V=U / 4$. The SDW and CDW order parameters do not vanish at the phase boundary and the transition is discontinuous. However, for finite values of $t^{\prime}$ the OAF and SN states which are energetically degenerate within the HF approximation, are found to be lower in energy than the CDW and SDW states over a finite portion of the phase diagram, whose size grows with increasing $t^{\prime}$.

Our main findings (summarized in Fig.2) indicate that the absence of nesting (which is destroyed by a finite $t^{\prime}$ ) favours the occurence of the OAF and SN states. This is significant since most real materials have non-nested Fermi surfaces. Stable OAF and SN states are found to occur in the weak correlation limit ( $U, V$ less than the bandwidth) where the HF approximation is expected to be reliable. The d-wave character of the particle-hole binding involved in the formation of the OAF and SN states, is interesting in the light of the observation of a pseudo-gap with a d-wave symmetry in photoemission experiments [9, 10] in the underdoped cuprates which has been interpreted variously as evidence for a paired state where superconductivity has been destroyed by phase fluctuations [11] or a manifestation of the spinon dispersion predicted by slave boson theories of the t-J model [12]. It is worth pointing out that d-wave pairing in the particle-hole channel, such as occurs in the OAF and SN phases, also leads to a similar one-particle excitation spectrum which is qualitatively consistent with the photoemission data.

We consider the extended Hubbard model on a square lattice, whose Hamiltonian is given by

$$
H=\sum_{i, j, \sigma} t_{i j} c_{i, \sigma}^{\dagger} c_{j, \sigma}-\mu \sum_{i, \sigma} \hat{n}_{i, \sigma}+U \sum_{i} \hat{n}_{i, \uparrow} \hat{n}_{i, \downarrow}+\frac{V}{2} \sum_{i, \sigma, \sigma^{\prime}} \sum_{\delta_{n n}} \hat{n}_{i, \sigma} \hat{n}_{i+\delta_{n n}, \sigma^{\prime}}
$$

The hopping matrix elements $t_{i j}$ are chosen to be $t_{i j}=-t$ for $i, j$ nearest-neighbours, $t_{i j}=t^{\prime}$ for $i, j$ next nearest neighbours and zero otherwise. Here $\mu$ is the chemical potential and $U$ and $V$ are the onsite and nearest neighbour repulsive interactions respectively. We work throughout at half-filling with one electron per site on the average. We consider mean-field states characterized by an anomalous non-zero ground state expectation value of the average $\left\langle c_{\mathbf{k}, \alpha}^{\dagger} c_{\mathbf{k}+\mathbf{Q}, \beta}\right\rangle$ with $\mathbf{Q}=(\pi, \pi)$ being the nesting vector of the non-interacting Fermi surface for $t^{\prime}=0$. 
The CDW order parameter results from a real space decoupling of the form

$$
\left\langle\hat{n}_{i, \sigma}\right\rangle=\frac{1}{2}+\frac{p}{2} \cos \left(\mathbf{Q} \cdot \mathbf{R}_{i}\right)
$$

whereas the SDW state (we consider a z-polarized state) is described by

$$
\left\langle\hat{n}_{i, \sigma}\right\rangle=\frac{1}{2}+\sigma \frac{m}{2} \cos \left(\mathbf{Q} \cdot \mathbf{R}_{i}\right)
$$

$\sigma=+(-) 1$ for up (down) spins. These order parameters describe a periodic modulation in the charge and spin density respectively resulting in a doubling of the unit cell. $p$ and $m$ are the amplitudes of the induced density modulation.

The OAF state on the other hand has non-zero intersite averages of the form

$$
\left\langle c_{i, \sigma}^{\dagger} c_{i \pm \hat{x} \sigma}\right\rangle=\imath g \cos \left(\mathbf{Q} \cdot \mathbf{R}_{i}\right)
$$

and

$$
\left\langle c_{i, \sigma}^{\dagger} c_{i \pm \hat{y} \sigma}\right\rangle=-\imath g \cos \left(\mathbf{Q} \cdot \mathbf{R}_{i}\right)
$$

The SN state (we consider a z-polarised state) has

$$
\left\langle c_{i, \sigma}^{\dagger} c_{i \pm \hat{x} \sigma}\right\rangle=\imath \ell \sigma \cos \left(\mathbf{Q} \cdot \mathbf{R}_{i}\right)
$$

and

$$
\left\langle c_{i, \sigma}^{\dagger} c_{i \pm \hat{y} \sigma}\right\rangle=-\imath \ell \sigma \cos \left(\mathbf{Q} \cdot \mathbf{R}_{i}\right)
$$

It is easy to see from Eqns.(3) and (4) that the OAF and SN states have charge and spin currents (whose magnitude is proportional to $g$ and $l$ respectively) moving along the elementary square plaquettes of the lattice with neighbouring plaquettes having their currents in opposite directions (see Fig.1). These states have been discussed earlier by several workers [3-6]. However, their stability with respect to the CDW and SDW states has never been precisely established. Earlier calculations [3-5] have approximately evaluated the appropriate RPA susceptibilities in the normal state. The divergence of these susceptibilities gives the mean field transition and the temperature where this happens is the mean field transition temperature. It is well-known 13 that in two dimensions, enhanced fluctuations prevent long range order from developing at any finite temperature. The RPA fails to take proper account of these enhanced fluctuations and thus predicts a spurious finite temperature phase transition. The system continues to be a Fermi liquid below the mean field transition temperature. However, this temperature is 
a crossover scale below which the Fermi liquid begins to develop short range correlations appropriate to the type of broken symmetry considered. The corresponding correlation length increases as the temperature is reduced and diverges at $T=0$. A comparision of the mean field transition temperatures of the different states is a rough indication of their relative stability. However, an accurate determination of the zero temperature phase diagram of the model Hamiltonian requires a complete solution of the self-consistent equations (obtained by minimizing the ground state energy) for the chemical potential and the order parameter. A comparision of the corresponding ground state energies then fixes the stable ground state for the parameters considered. The resulting phase diagram is the main result of this paper.

We first obtain the self-consistent equations for the order parameter and the chemical potential (which has to be determined self-consistently for $t^{\prime} \neq 0$ ). On substituting the mean field ansatz of $\operatorname{Eqns}(2-4)$ in the Hamiltonian of $\operatorname{Eqn}(1)$ we find that the mean field Hamiltonian, for the various states considered, is given by

$$
\begin{aligned}
H_{M F}^{s}= & \sum_{\mathbf{k}, \alpha}^{R B Z}\left[\left(\xi_{\mathbf{k}}-\mu\right) c_{\mathbf{k}, \alpha}^{\dagger} c_{\mathbf{k}, \alpha}+\left(\xi_{\mathbf{k}+\mathbf{Q}}-\mu\right) c_{\mathbf{k}+\mathbf{Q}, \alpha}^{\dagger} c_{\mathbf{k}+\mathbf{Q}, \alpha}\right] \\
& +\sum_{\mathbf{k}, \alpha, \beta}^{R B Z}\left[\left(\Delta_{\alpha, \beta}^{s}(\mathbf{k}) c_{\mathbf{k}, \alpha}^{\dagger} c_{\mathbf{k}+\mathbf{Q}, \beta}+\text { h.c }\right]+X_{s}\right.
\end{aligned}
$$

The reduced Brillouin zone (RBZ) is formed due to the modulation with wavevector Q, which introduces a two-sublattice structure resulting in a folding up of the original Brillouin zone. The tight-binding dispersion is $\xi_{\mathbf{k}}=-2 t\left[\cos k_{x}+\cos k_{y}\right]+4 t^{\prime} \cos k_{x} \cos k_{y}$ and $\Delta_{\alpha, \beta}^{s}(\mathbf{k})$ is the generalized order parameter where $s=1,2,3, \& 4$ correspond to CDW, SDW, OAF and SN states respectively. Thus,

$$
\begin{array}{lr}
\Delta_{\alpha, \beta}^{1}(\mathbf{k})=\delta_{\alpha, \beta}\left(\frac{U}{2}-4 V\right) p & \text { for the CDW state }(6 a) \\
\Delta_{\alpha, \beta}^{2}(\mathbf{k})=\sigma_{\alpha, \beta}^{z}\left(-\frac{U}{2}\right) m & \text { for the } \mathrm{z}-\text { polarized SDW state }(6 b) \\
\Delta_{\alpha, \beta}^{3}(\mathbf{k})=\imath \delta_{\alpha, \beta}\left(\cos k_{x}-\cos k_{y}\right)(-2 V) g & \text { for the OAF state }(6 c)
\end{array}
$$

and $\quad \Delta_{\alpha, \beta}^{4}(\mathbf{k})=\imath \sigma_{\alpha, \beta}^{z}\left(\cos k_{x}-\cos k_{y}\right)(-2 V) \ell \quad$ for the $\mathrm{z}-$ polarized SN state $(6 d)$ where $\sigma^{z}$ is a Pauli matrix. In writing Eqn(5) we have ignored the Hartree contribution $(U / 2+4 V)$ which shifts the energy of each state by an equal amount and can therefore 
be absorbed in the chemical potential. The constant piece $X_{s}$ in the respective ground state energies is given by

$$
\begin{aligned}
X_{1} & =\left(2 V-\frac{U}{4}\right) p^{2} N \\
X_{2} & =\frac{U}{4} m^{2} N \\
X_{3} & =4 V g^{2} N \\
X_{4} & =4 V \ell^{2} N
\end{aligned}
$$

where $\mathrm{N}$ is the total number of sites. In writing $\operatorname{Eqn}(7)$ we have once again ignored the Hartree contribution of $-(U / 4+2 V) N$ which merely shifts the ground-state energies of each of the mean field solutions by an equal amount.

The mean field Hamiltonian of Eqn(5) is quadratic and can be diagonalized by a canonical transformation. The folding of the Brillouin zone, caused by the $(\pi, \pi)$ modulation, results in the formation of two bands whose dispersion is given by

$$
e_{s}^{(+)}(\mathbf{k})=\left(\eta_{\mathbf{k}}-\mu\right)+\sqrt{\epsilon_{\mathbf{k}}^{2}+\Delta_{s}^{2}(\mathbf{k})}
$$

and

$$
e_{s}^{(-)}(\mathbf{k})=\left(\eta_{\mathbf{k}}-\mu\right)-\sqrt{\epsilon_{\mathbf{k}}^{2}+\Delta_{s}^{2}(\mathbf{k})}
$$

Here $\Delta_{1}(\mathbf{k})=(U / 2-4 V) p, \Delta_{2}(\mathbf{k})=-U m / 2, \Delta_{3}(\mathbf{k})=-2 V g\left(\cos k_{x}-\cos k_{y}\right)$ and $\Delta_{4}(\mathbf{k})=-2 V \ell\left(\cos k_{x}-\cos k_{y}\right), \eta_{\mathbf{k}}=\left(\xi_{\mathbf{k}}+\xi_{\mathbf{k}+\mathbf{Q}}\right) / 2, \epsilon_{\mathbf{k}}=\left(\xi_{\mathbf{k}}-\xi_{\mathbf{k}+\mathbf{Q}}\right) / 2$ and $\xi_{\mathbf{k}}$ is the band dispersion in the non interacting limit. For $t^{\prime}=0$, nesting of the Fermi surface ensures that $\eta_{\mathbf{k}}=0$. Then, the chemical potential stays pinned to it's non-interacting value $(\mu=0)$ and the lower band $\left(e_{\mathbf{k}}^{(-)}\right)$is completely full whereas the upper band is completely empty. However when $t^{\prime}$ becomes finite, $\eta_{\mathbf{k}} \neq 0$ and the bands begin to overlap. It is easy to see that CDW $\left(\Delta_{1}(\mathbf{k})\right)$ and SDW $\left(\Delta_{2}(\mathbf{k})\right)$ states are isotropic (swave) whereas the OAF and SN states $\left(\Delta_{3}(\mathbf{k})\right.$ and $\left.\left.\Delta_{4}(\mathbf{k})\right)\right)$ states have order parameters of $d_{x^{2}-y^{2}}$ character.

Using the diagonalized form of the Hamiltonian, we obtain the self-consistent equations for the chemical potential $\mu$ and the order parameters, which are given by

$$
\frac{1}{2}=\frac{1}{N} \sum_{\mathbf{k}}^{R B Z}\left[\Theta\left(-e_{s}^{(-)}(\mathbf{k})\right)+\Theta\left(-e_{s}^{(+)}(\mathbf{k})\right)\right]
$$


and

$$
\frac{1}{\Gamma_{s}}=\frac{1}{N} \sum_{\mathbf{k}}^{R B Z} \frac{w_{\mathbf{k}}^{s}\left[\Theta\left(-e_{s}^{(-)}(\mathbf{k})\right)+\Theta\left(-e_{s}^{(+)}(\mathbf{k})\right)\right]}{\sqrt{\epsilon_{\mathbf{k}}^{2}+\Delta_{s}^{2}(\mathbf{k})}}
$$

Here $\Gamma_{1}=4 V-U / 2, \Gamma_{2}=U / 2, \Gamma_{3}=\Gamma_{4}=V / 2$ and $w_{\mathbf{k}}^{s}$ is the symmetry factor of the order parameters with $w_{\mathbf{k}}^{1}=w_{\mathbf{k}}^{2}=1\left(\right.$ for SDW \& CDW) and $w_{\mathbf{k}}^{3}=w_{\mathbf{k}}^{4}=\left(\cos k_{x}-\cos k_{y}\right)^{2}$ (for OAF \& SN).

We have solved Eqn(9) numerically to determine the self-consistent values of the chemical potential $\mu$ and the gap function $\Delta_{s}(\mathbf{k})$. Self-consistent solutions for all the four mean field states exist for almost all values of $U / t$ and $V / t$ and therefore it becomes necessary to compare the ground state energies in order to decide the relative stabilities of these states. The ground state energy of a given state can be easily found from the diagonalized Hamiltonian and is given by

$$
E^{s}=X_{s}+\frac{2}{N} \sum_{k}^{R B Z}\left[e_{s}^{(-)}(\mathbf{k}) \Theta\left(-e_{s}^{(-)}(\mathbf{k})\right)+e_{s}^{(+)}(\mathbf{k}) \Theta\left(-e_{s}^{(+)}(\mathbf{k})\right)\right]
$$

It is easy to see from a comparison of $\operatorname{Eqns}(9)$ and (10) that the OAF and SN states are degenerate within the Hartree-Fock approximation for our model. Further, the dispersion of the energy bands $\left(e^{(-)}(\mathbf{k})\right.$ and $\left.e^{(+)}(\mathbf{k})\right)$ is also identical. These states are very different physically. The OAF state has a staggered orbital magnetic moment produced by the circulating charge currents which should be observable in magnetic neutron scattering experiments, whereas the spin-currents present in the SN state would not directly couple to the neutron magnetic moment. However, they are identical in their one-particle properties as far as experiments which probe their band structure are concerned.

We use the self-consistent values of $\Delta_{s}(\mathbf{k})$ and $\mu$, obtained from the solution of $\operatorname{Eqn}(9)$, to evaluate the ground state energy $E_{s}$ in $\operatorname{Eqn}(10)$ for different parameter values $t^{\prime} / t, U / t$ and $V / t$. For $t^{\prime}=0$, we find that the CDW and SDW states are the only stable states and are separated by a phase boundary at $U=4 V$. In Fig.2 we present the phase diagram obtained from $\operatorname{Eqns}(9)$ and (10) for typical values of $t^{\prime} / t$ in the $(U / t, V / t)$ plane. Our results indicate that the destruction of nesting, introduced by $t^{\prime}$ helps to stabilize the $\mathrm{OAF} / \mathrm{SN}$ states at weak coupling. The various order parameters do not vanish at the phase boundaries which therefore indicate discontinuous phase transitions. The phase boundaries for small values of the interaction parameters $U / t$ and $V / t$ have not been shown as numerical difficulties prevented an accurate determination of their positions. 
The zero temperature instability of the Fermi system to the states considered by us can be roughly understood as arising from the energy gained due to the opening up of a gap $\Delta(\mathbf{k})$ in the energy spectrum which pushes down (in energy) the occupied states. It is then easy to see from Eqn. (6) that the gap functions in the CDW, SDW and OAF/SN states are proportional to $4 V-U / 2, U / 2$ and $2 V$ respectively. Thus the OAF/SN states are expected to be energetically close to the other two states near the $U=4 V$ line. At large values of $U$, the dominant consideration is to minimize the double occupancy in the ground state which is achieved by the SDW state with $m \rightarrow 1$. On the other hand for large values of $V$, the dominant tendency is for all the electrons to sit on one of the sublattices thus completely avoiding the energy cost associated with having electrons on neighbouring sites. This corresponds to the CDW state with $p \rightarrow 1$. Thus the OAF/SN states are expected to arise only at weak coupling and close to the $U=4 \mathrm{~V}$ line. This is borne out by our numerical results. The crucial role of $t^{\prime}$ in stabilizing the OAF/SN states is apparent from our phase diagram. The physical basis for this is not clear to us. However it suggests that the $\mathrm{OAF} / \mathrm{SN}$ states are more robust with respect to the destruction of the nesting of the Fermi surface than the CDW or the SDW states.

In Fig.3 we show the dispersion $e_{\mathbf{k}}^{(-)}$and $e_{\mathbf{k}}^{(+)}$for the OAF (SN) states as a function of $\mathbf{k}$ for $t^{\prime} / t$ finite with $U / t$ and $V / t$ chosen to ensure the stability of the phases considered. It is clear from Fig. 3 that for $t^{\prime} \neq 0$ the upper and lower bands overlap. Thus the valence band is not completely full and the conduction band is not completely empty. The OAF (SN) state is therefore a poor metallic state with a pseudogap at the Fermi surface.

Finally, we summarize the main results of this paper. We have obtained the zero temperature phase diagram of the extended Hubbard model on a square lattice at halffilling. We find that for finite values of next-nearest-neighbour hopping amplitude $t^{\prime}$, the OAF/SN states have lower ground state energy than the SDW and CDW state over a finite range of parameters in the weak coupling region. The size of this region, where $\mathrm{OAF} / \mathrm{SN}$ states are stable, increases with increasing $t^{\prime}$.

We would like to thank A. Taraphder, R. Pandit and T. V. Ramakrishnan for useful discussions. 


\section{References}

[1] J. R. Schrieffer, X. G. Wen and S. C. Zhang, Phys. Rev. B 39, 11663 (1989).

[2] B. I. Halperin and T. M. Rice, Solid State Physics, Vol. 21, ed. F. Seitz, D. Turnbull and H. Ehrenreich, Academic Press, New York (1968).

[3] H. J. Shulz, Phys. Rev. B 39, 2940 (1989).

[4] A. A. Nersesyan and G. E. Vachnadze, J. Low Temp. Phys. 77, 293 (1989).

[5] A. A. Nersesyan, G. I. Japaridze and I. G. Kimeridze, J. Phys. Cond. Matt. 3, 3353 (1991).

[6] L. P. Gorkov and A. Sokol, Phys. Rev. Lett. 69, 2586 (1992).

[7] C. Nayak and F. Wilczek, Preprint, cond-mat/9510132

[8] I. Affleck and B. Marston, Phys. Rev. B 37, 3774 (1988).

[9] D. S. Marshall et. al., Phys. Rev. Lett. 76, 4841 (1996); A. G. Loesen et. al., Science 273, 325 (1996).

[10] H. Ding et. al, Nature 382, 51 (1996); Preprint, cond-mat/9611194.

[11] V. J. Emery and S. A. Kivelson, Nature 374, 434 (1995).

[12] R. B. Laughlin, Preprint, 5upr-con/9608005.

[13] N. D. Mermin, J. of Phys. Soc. Japan 26, Supplement, 203 (1969) 


\section{FIGURE CAPTIONS}

Fig.1. Schematic diagram of the circulating currents associated with the OAF and SN states. The arrows indicate the directions of the currents which are staggered and move oppositely on neighbouring plaquettes.

Fig.2. The mean-field phase diagram of the $t-t^{\prime}$ extended Hubbard model. All the phase boundaries indicate discontinuous transitions. The region of stability of the OAF $(\mathrm{SN})$ state grows with increasing $t^{\prime} / t$ ratio, as seen in the figure.

Fig.3. Plot of the quasiparticle dispersion energy $e_{\mathbf{k}}^{(+)}$and $e_{\mathbf{k}}^{(-)}$for the OAF and SN states along the symmetry directions in the reduced Brillouin zone. The solid curve indicates the conduction band dispersion while the dashed curve is for the valence band dispersion. The parameters choosen for the quasiparticle dispersion are $t^{\prime} / t=0.1, U / t=1.5$ and $V / t=0.37$ for which the OAF (SN) state is stable. Inset shows the position of the points $\Gamma, X$ and $\Sigma$ in the Brillouin zone. 